\title{
Metallic ferromagnetism in a generalized Hubbard model
}

\author{
L. Didukh and O. Kramar \\ Ternopil State Technical University, Department of Physics, \\ 56 Rus'ka Str., Ternopil 46001, Ukraine \\ E-mail: didukh@tu.edu.te.ua
}

Received June 20, 2001, revised July 16, 2001

\begin{abstract}
The possibility of realization of a metallic ferromagnetic state in a generalized Hubbard model with correlated hopping and exchange interaction integrals is investigated. The single-electron energy spectrum recently obtained by means of the mean-field approximation is applied for the description of ground-state and finite-temperature properties of the system. An expression for the Curie temperature is found, and the behavior of the temperature dependences of the magnetization and paramagnetic susceptibility is analyzed. Taking into account the correlated hopping allows to explain certain peculiarities of the ferromagnetic behavior of transition metals and their compounds.
\end{abstract}

PACS: 75.10.-b, 75.20.En, 75.30.Cr, 75.50.Bb, 75.50.Cc

\section{Introduction}

An important problem in the explanation of ferromagnetism in a single band of electrons is that of correctly taking into account the Coulomb correlations between electrons. The Hubbard model $[1,2,3]$, which describes a single nondegenerate electron band with local Coulomb interaction, is oversimplified and requires generalization. It is natural to generalize the Hubbard Hamiltonian by taking into account other matrix elements of electron-electron interaction (in addition to the intraatomic Coulomb repulsion) and to consider the ferromagnetism in the generalized Hubbard model (for review on this problem see Refs. 4-6).

Note that the problem of metallic ferromagnetism in the single-band Hubbard model and its generalizations has attracted much attention in a series of papers employing the dynamical mean-field theory $[7,8]$, the Gutzwilier variational wave function approximation $[9,10]$, the spectral density approximation $[11,12]$, the exact diagonalization method [13], and mean-field theory [14-16]. In the papers of Hirsch [14-16] a generalization of Stoner-Wolfarth theory $[17,18]$ was carried out. The Stoner-Wolfarth theory has been used for the description of itinerant electron magnetism (note in this connection that the calculations in this theory essentially depend on the shape and peculiarities of the density of states; in particular, it is known that the incomplete ferromagnetism in this model is absent if the density of states is rectangular [18]). This generalization [14-16] has shown that the inter-atomic exchange interaction plays an important role for obtaining a ferromagnetic state with partial polarization. Using the mean-field theory, Hirsch has obtained the condition of ferromagnetic state realization, nonmonotonic behavior of the concentration dependence of the magnetization, an expression for the Curie temperature, and the temperature dependences of the magnetization and magnetic susceptibility. Using a local approximation developed from the Gutzviller wave function method, the authors of Ref. 10 suggest also (in agreement with [14]) the importance of the interatomic exchange interaction for stabilization of the ferromagnetic state with partial spin polarization. In Ref. 19 the case of strong correlation was studied and the criterion of ferromagnetism and magnetization of the system in the ground state was derived; in this paper the importance of inter-atomic exchange interaction is also emphasized.

At the same time, from our point of view it is in principle necessary to consider (in addition to the inter-atomic exchange interaction) the matrix ele- 
ments which describe the correlated hopping of electrons [20] for the explanation of ferromagnetism in a single-band model. In this model an electron hopping from one site to another is correlated both by the occupation of the sites involved in the hopping process and the occupation of the nearest-neighbor sites. Taking into account the correlated hopping allows to obtain an additional mechanism $[21,22]$ for the stabilization of ferromagnetic ordering.

The importance of correlated hopping for understanding of metallic ferromagnetism was discussed in [13], where the results obtained by means of exact diagonalization for small one-dimensional chains were compared with mean-field theory results. It has been shown that in the strong-coupling regime, correlated hopping favors ferromagnetism stronger for electron concentration $n>1$ than for $n<1$ (the reverse situation occurs for weak interactions [13]); this result agrees with conclusions of [23]. Using the Gutzviller approach the authors of [9] have shown that the correlated hopping strongly favors the ferromagnetic ordering close to the point of half filling.

The magnetic properties of the system at nonzero temperature were analyzed in a series of papers $[7,8,11,12]$. However, the important question concerning the influence of correlated hopping on the Curie temperature and on the behavior of the magnetization and magnetic susceptibility at nonzero temperature has not yet been considered.

In this paper the theory of metallic ferromagnetism in a model which includes the intra-atomic Coulomb interaction, the interatomic exchange interaction of electrons, and the electron-electron interaction due to electron hopping (correlated hopping) is formulated. The application of the meanfield approximation to this generalized Hubbard model with correlated hopping leads to a correct description of ferromagnetism for zero temperature (in this case our results agree with the results obtained by Hirsch in $[14,15])$ and also can eliminate the problem of overestimation of the Curie temperature (in consequence of taking into account the correlated hopping). Besides, the mean-field analysis of this model, in spite of its limitations, allows one to reproduce the behavior of the magnetic moment and Curie temperature with changing electron concentration and obtain the correct temperature dependence of the magnetic moment of the system. Note that the mean-field treatment of models which include only the band part of the Hamiltonian and intra-atomic Coulomb interaction (or exchange interaction) predicts values for the Curie temperature which are larger than those observed in transition metals by an order of magnitude.

The paper is organized as follows. In Sec. 2 the Hamiltonian of the generalized Hubbard model with correlated hopping and inter-atomic exchange interaction is written, and the single-electron energy spectrum obtained by means of the Green function technique is analyzed. In Sec. 3 the ground-state properties of the system are considered, the equation for the system critical parameters and the expression for the magnetization are derived. In Sec. 4 the finite-temperature properties of the system are considered, and expression for the Curie temperature and the temperature dependences of the magnetization and magnetic susceptibility are obtained. Sec. 5 is devoted to the conclusions.

\section{The model Hamiltonian and single-electron energy spectrum}

Consider the Hamiltonian proposed in [24], generalized by taking into account a weak magnetic field:

$$
\begin{gathered}
H=-\mu \sum_{i \sigma} a_{i \sigma}^{+} a_{i \sigma}+\sum_{i j \sigma}^{\prime} t_{i j}(n) a_{i \sigma}^{+} a_{j \sigma}+ \\
+\sum_{i j \sigma}^{\prime}\left(T_{2}(i j) a_{i \sigma}^{+} a_{j \sigma} n_{i \bar{\sigma}}+\text { h.c. }\right)+U \sum_{i} n_{i \uparrow} n_{i \downarrow}+ \\
+\frac{J}{2} \sum_{i j \sigma \sigma^{\prime}}^{\prime} a_{i \sigma^{+}}^{+} a_{j \sigma^{\prime}}^{+} a_{i \sigma^{\prime}} a_{j \sigma}-h \sum_{i}\left(n_{i \uparrow}-n_{i \downarrow}\right),
\end{gathered}
$$

where $a_{i \sigma}^{+}, a_{i \sigma}$ are the creation and annihilation operators of electron on site $i, \sigma=\uparrow, \downarrow, n_{i \sigma}=a_{i \sigma}^{+} a_{i \sigma}$ is the operator of number of electrons with spin $\sigma$ on site $i, n=\left\langle n_{i \uparrow}+n_{i \downarrow}\right\rangle, \mu$ is the chemical potential, $t_{i j}(n)=t_{i j}+n T_{1}(i j)$ is the effective integral of an electron hopping from site $j$ to site $i, t_{i j}$ is the band hopping integral of an electron from site $j$ to site $i$, $T_{1}(i j)$ and $T_{2}(i j)$ are the parameters of correlated hopping of electrons, $U$ is the intra-atomic Coulomb repulsion, $J$ is the exchange integral for the nearest neighbors, and $h$ is the external magnetic field (the units of $h$ are such that the magnetic moment per electron is unity). The prime on the sums in Eq. (1) signifies that $i \neq j$. The concentration dependence of the effective hopping integral $t_{i j}(n)$ is caused by the correlated hopping [24] of electrons.

The peculiarities of the model described by Hamiltonian (1) are the taking into account of the influence of site occupation on hopping process (correlated hopping) and the direct exchange inter- 
action between electrons on the neighboring sites. To characterize the value of correlated hopping we introduce dimensionless parameters $\tau_{1}=T_{1}(i j) /\left|t_{i j}\right|$, $\tau_{2}=T_{2}(i j) /\left|t_{i j}\right|$ which are independent on the number of site.

Using the mean-field approximation in the Green function method, we have recently obtained [22] the single-electron energy spectrum. In an external magnetic field it has the form

$$
E_{\sigma}(\mathbf{k})=-\mu+\beta_{\sigma}+n_{\sigma} U-z n_{\sigma} J-h \eta_{\sigma}+t(n \sigma) \gamma(\mathbf{k}) ;
$$

here the spin-dependent shift of the band centers is

$$
\beta_{\sigma}=\frac{2}{N} \sum_{i j} T_{2}(i j)\left\langle a_{i \bar{\sigma}}^{+} a_{i \bar{\sigma}}\right\rangle,
$$

$z$ is the number of nearest neighbors to a site, for the spin $\sigma=\uparrow(\downarrow)$ we have $\eta_{\sigma}=1(-1), \quad \gamma(\mathbf{k})=$ $=\sum_{R} \mathrm{e}^{i \mathbf{k R}}$ (the sum goes over the nearest neighbors to a site), and the spin- and concentration-dependent hopping integral is

$$
t(n \sigma)=\left(1-\tau_{1} n-2 \tau_{2} n_{\bar{\sigma}}-\frac{z J}{w} \sum_{\sigma^{\prime}} A_{\sigma^{\prime}}\right) t=\alpha_{\sigma} t,
$$

where $w=z|t|$ is half of bandwidth, $t$ is the hopping integral between nearest-neighbour sites, and

$$
A_{\sigma^{\prime}}=\frac{1}{N} \sum_{i j}\left(-\frac{t_{i j}}{w}\left\langle a_{i \sigma^{\prime}}^{+} a_{j \sigma^{\prime}}\right\rangle\right) .
$$

Energy spectrum (2) will be used in the next Sections for the description of the model properties in the ground state and at finite temperature.

\section{The ground-state properties of the model}

The concentration of electrons with spin $\sigma$ is

$$
n_{\sigma}=\int_{-w}^{w} \rho(\varepsilon) f\left(E_{\sigma}(\varepsilon)\right) d \varepsilon
$$

where $\rho(\varepsilon)$ is the density of states, and $f\left(E_{\sigma}(\varepsilon)\right)$ is the Fermi distribution function. Thus the occupation number and the magnetization are expressed, respectively, as

$$
\begin{gathered}
n=n_{\uparrow}+n_{\downarrow}=\int_{-w}^{w} \rho(\varepsilon)\left[f\left(E_{\uparrow}(\varepsilon)\right)+f\left(E_{\downarrow}(\varepsilon)\right)\right] d \varepsilon ; \\
m=n_{\uparrow}-n_{\downarrow}=\int_{-w}^{w} \rho(\varepsilon)\left[f\left(E_{\uparrow}(\varepsilon)\right)-f\left(E_{\downarrow}(\varepsilon)\right)\right] d \varepsilon .
\end{gathered}
$$

Let us assume the unperturbed rectangular density of states $\rho(\varepsilon)=\Theta\left(\varepsilon^{2}-w^{2}\right) / 2 w$. In the case of zero temperature one can obtain for the correlation function $A_{\sigma}$ and the shift $\beta_{\sigma}$ of the center of the $\sigma$ subband

$$
\begin{gathered}
A_{\sigma}=n_{\sigma}\left(1-n_{\sigma}\right), \\
\beta_{\sigma}=2 w \tau_{2} A_{\sigma}=2 w \tau_{2} n_{\sigma}\left(1-n_{\sigma}\right),
\end{gathered}
$$

where the concentration of electrons with spin $\sigma$

$$
n_{\sigma}=\frac{\varepsilon_{\sigma}+w}{2 w}
$$

here $\varepsilon_{\sigma}=\mu_{\sigma} / \alpha_{\sigma}$ is the solution of the equation $E_{\sigma}(\varepsilon)=0$, where $\mu_{\sigma}=\mu-\beta_{\sigma}+z n_{\sigma} J-n_{\sigma} U+h \eta_{\sigma}$ and $\alpha_{\sigma}=1-\tau_{1} n-2 \tau_{2} n_{\bar{\sigma}}-z J / w \sum_{\sigma^{\prime}} A_{\sigma^{\prime}}$.

On basis of expressions (7)-(8) using (9)-(10), one can obtain the equation for the system critical parameters:

$$
\begin{gathered}
\frac{z J}{2 w}\left[1+n(2-n)-m^{2}\right]= \\
=1-n \tau_{1}-\tau_{2}(2-n)-\frac{U}{2 w}-\frac{h}{w} .
\end{gathered}
$$

Expression (12), which determines the stability condition for ferromagnetism, agrees with the expresion obtained from an analysis of the groundstate energy [22]. The condition for onset of feromagnetic ordering is obtained (replacing the equals sign by an inequality sign) when $m=0$, and the condition for the ferromagnetic state with full spin polarization is derived by putting $m=n$ in expression (12). The peculiar distinction of our expression from the similar condition obtained in $[10,14-16]$ for the various generalizations of the Hubbard model is the presence of correlated hopping, which can substantially modify the properties of the model. Taking into account correlated hopping leads to the appearance of a peculiar kinetic mechanism of ferro- 
magnetic ordering stabilization. This mechanism is caused by the presence of the spin-dependent shift of the spin subband centers, which is a consequence of correlated hopping (it is analogous to the shift of subband centers in consequence of the interatomic direct exchange interaction).

The spontaneous ground-state magnetization (in the absence of magnetic field) of the system is found from Eq. (12) as

$m_{0}=\left[1+n(2-n)-\frac{1-U / 2 w-\tau_{1} n-\tau_{2}(2-n)}{z J / 2 w}\right]^{1 / 2}$,

which is valid in the case $J>0$ (when $J=0$ only the transition from the paramagnetic to the fully polarized ferromagnetic state with $m_{0}=n$ occurs). If the calculated magnetization $m_{0}>n$, then it is necesarry to put $m_{0}=n$.

The influence of correlated hopping on the properties of the system is illustrated in Figs. 1 and 2. In Fig. 1 the dependence of the critical value of the exchange integral at which the ferromagnetic ordering occurs is plotted (in the absence of magnetic field) as a function of band filling for various values of correlated hopping parameters $\tau_{1}, \tau_{2}$ and intraatomic Coulomb interaction $U / w$. The solid curves correspond to the onset of spin polarization and the dashed curves to the fully polarized ferromagnetic state (the area below the solid line is paramagnetic, above the dashed line - fully polarized ferromagnetic, between these lines - partially polarized ferromagnetic). Similar phase diagrams have been obtained in $[14,16]$, but in those works the corre-

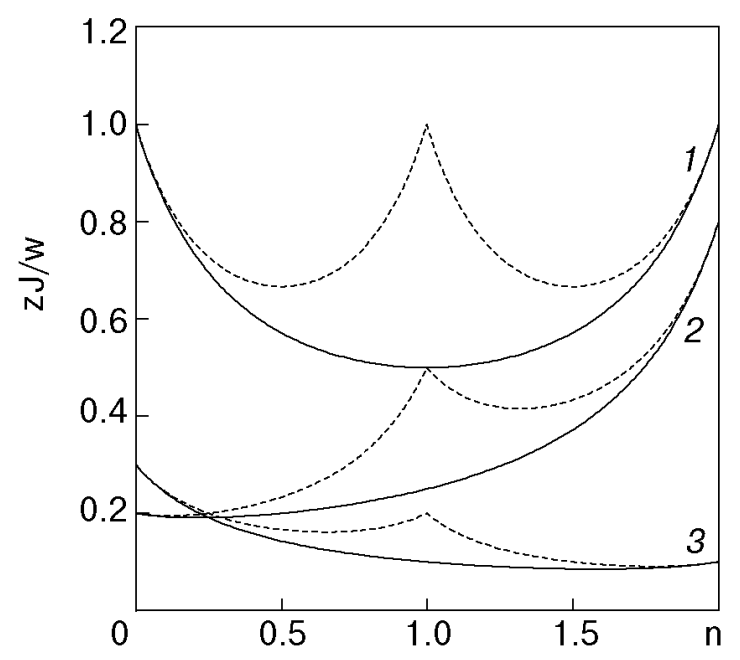

Fig. 1. Critical values of $z J / w$ as a function of $n$ at fixed value of $U / w . U / w=1, \tau_{1}=\tau_{2}=0$ (1); $U / w=$ $=1.2, \quad \tau_{1}=0, \quad \tau_{2}=0.15 \quad(2) ; \quad U / w=1.3, \quad \tau_{1}=0.15$, $\tau_{2}=0.1(3)$.

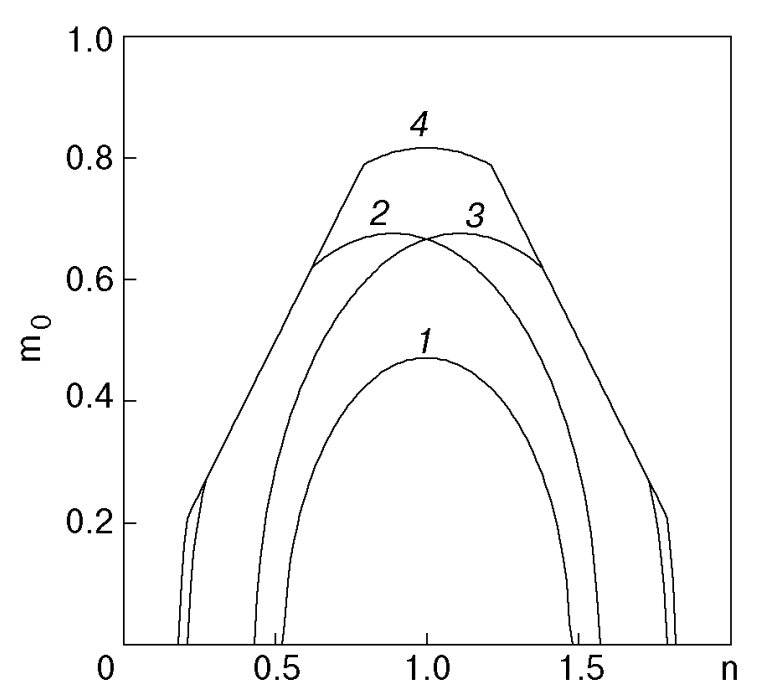

Fig. 2. Ground-state magnetization $m_{0}$ as a function of $n$ for $U / w=1.2$ and $z J / w=0.1 . \tau_{1}=\tau_{2}=0$ (1); $\tau_{1}=0$, $\tau_{2}=0.05(2) ; \tau_{1}=0.05, \tau_{2}=0$ (3); $\tau_{1}=\tau_{2}=0.05$ (4).

lated hopping was not considered. Note that the presence of correlated hopping (curves 2, 3 in Fig. 1) leads to a substantial change of the phase boundary of the paramagnet-ferromagnet transition; in particular, to a shift of the minimum point, namely to the inequivalence of the cases $n<1$ and $n>1$.

In Fig. 2 the dependence of the ground-state magnetization $m_{0}$ on the electron concentration $n$ for fixed values of exchange and Coulomb interactions, as well as for different values of correlated hopping parameters, is plotted. In Refs. 14, 16 the concentration dependences of the magnetization were obtained in the framework of the Hubbard model with interatomic exchange, but they are symmetric with respect to half-filling. In Ref. 19 a similar $m_{0}(n)$ dependence was obtained.

As mentioned above, asymmetry of the cases $n<1$ and $n>1$ is obsereved; in particular, an increase of parameter $\tau_{1}$ leads to a shift of the ferromagnetic area to a region of larger electron concentration $n$, while an increase of parameter $\tau_{2}$ gives a shift to smaller $n$. Note also that taking into account the correlated hopping enriches considerably the set of curves $m_{0}(n)$. The obtained concentration dependences of the magnetization allow one to describe qualitatively the experimental curves for the binary ferromagnetic alloys of transition metals Fe, Co, Ni (Slater-Pouling's curves [26]). 


\section{The properties of the model at nonzero temperature}

For nonzero temperature and unperturbed rectangular density of states the concentration of electrons with spin $\sigma$ is expressed from (6) as

$$
n_{\sigma}=1-\frac{\Theta}{2 w \alpha_{\sigma}} \ln \left[\frac{1+\exp \left(\frac{E_{\sigma}(w)}{\Theta}\right)}{1+\exp \left(\frac{E_{\sigma}(-w)}{\Theta}\right)}\right] .
$$

Using expression (8) one can obtain the equation for the magnetization

$$
\exp \left(-\frac{m J_{\mathrm{eff}}}{\Theta}\right)=\frac{\sinh \left(\frac{\left(1-n_{\uparrow}\right) \alpha_{\uparrow} w}{\Theta}\right) \sinh \left(\frac{n_{\downarrow} \alpha_{\downarrow} w}{\Theta}\right)}{\sinh \left(\frac{\left(1-n_{\downarrow}\right) \alpha_{\downarrow} w}{\Theta}\right) \sinh \left(\frac{n_{\uparrow} \alpha_{\uparrow} w}{\Theta}\right)},
$$

where $J_{\text {eff }}=z J+U+2 \tau_{2} w(1-n)$. To obtain the temperature dependence of the magnetization it is necessary to apply numerical methods inasmuch as the last equation can not be solved analytically. The numerical calculations show that the results could be approximately expressed using the approach proposed in [15]. The Curie temperature can be obtained by expanding (8) to lowest order in $m \rightarrow 0$ :

$$
\begin{gathered}
1=\frac{1}{2 w} \frac{U+z J-2 w \tau_{2}(n-1)}{\alpha^{*}} \int_{-w \alpha^{*}}^{w \alpha^{*}}\left[-\frac{\partial f\left(x-\mu^{*}\right)}{\partial x}\right] d x- \\
-\frac{\tau_{2}}{w\left(\alpha^{*}\right)^{2}} \int_{-w \alpha^{*}}\left[-\frac{\partial f\left(x-\mu^{*}\right)}{\partial x}\right] x d x
\end{gathered}
$$

here $x=\alpha^{*} \varepsilon, \alpha^{*}=\left.\alpha_{\sigma}\right|_{m=0}$ and $\mu^{*}=\left.\mu_{\sigma}\right|_{m=0}$.

At low temperature one can approximately write

$$
1=\frac{U / 2 w+z J / 2 w-2 w \tau_{2}(n-1)}{1-\left(\tau_{1}+\tau_{2}\right) n-2 A^{*}\left(\Theta_{C}\right) z J / w},
$$

where $A^{*}\left(\Theta_{C}\right)=\left.A_{\sigma}(\Theta)\right|_{m=0}, \Theta=k_{B} T, \quad k_{B}$ is the Boltzmann constant. In the case of zero temperature the last expression reproduces the criterion of the paramagnetic-ferromagnetic transition obtained in a recent paper [22].

To find $A_{\sigma}(\Theta)$, as in the paper [15], one can apply the Sommerfeld expansion [25]

$$
\begin{aligned}
& A_{\sigma}(\Theta)=\int_{-w}^{\omega}\left[-\frac{\varepsilon}{2 \omega^{2}}\right] \frac{d \varepsilon}{\exp \left(E_{\sigma}(\varepsilon) / \Theta\right)+1} \simeq \\
& \simeq n_{\sigma}\left(1-n_{\sigma}\right)-\frac{\pi^{2}}{3}\left(\frac{\Theta}{2 w}\right)^{2} \times \\
& \times \frac{1}{\left[1-\tau_{1} n-2 \tau n_{\bar{\sigma}}-\left(A_{\uparrow}(\Theta)+A_{\downarrow}(\Theta)\right) z J / \omega\right]^{2}} .
\end{aligned}
$$

In the Curie point (when the magnetic moment $m \rightarrow 0$ and $\left.n_{\sigma}=n / 2\right)$ the last equation is written as

$$
\begin{gathered}
A^{*}\left(\Theta_{C}\right)=\frac{n(2-n)}{4}-\frac{2 \pi^{2}}{3}\left(\frac{\Theta_{C}}{2 w}\right)^{2} \times \\
\frac{1}{\left[1-\left(\tau_{1}+\tau_{2}\right) n-2 A^{*}\left(\Theta_{C}\right) z J / \omega\right]^{2}} .
\end{gathered}
$$

Solving the system of equations (17) and (19), we can express the Curie temperature as a function of the model parameters as

$$
\begin{aligned}
\frac{\Theta_{C}}{2 w}=[1+ & \left.n(2-n)-\frac{1-U / 2 w-\tau_{1} n-\tau_{2}(2-n)}{z J / 2 w}\right]^{1 / 2} \times \\
& \times\left(\frac{3}{2 \pi^{2}}\right)^{1 / 2}\left[\frac{U}{2 w}+\frac{z J}{2 w}-2 \tau_{2}(n-1)\right] .
\end{aligned}
$$

Taking into account expression (13), we finally obtain for the Curie temperature

$$
\frac{\Theta_{C}}{2 \omega}=\left(\frac{3}{2 \pi^{2}}\right)^{1 / 2}\left[\frac{U}{2 \omega}+\frac{z J}{2 w}-2 \tau_{2}(n-1)\right] m_{0} .
$$

In the absence of correlated hopping, formula (21) reproduces the result of Ref. 15. Note that the Curie temperature is closely related (in the approximation used) to the ground state magnetization of the ferromagnet. The peculiarity of formula (21) is the presence of the term containing the correlated hopping parameter $\tau_{2}\left(m_{0}\right.$ is also dependent on this parameter), which in the case $n>1$ is negative and therefore lowers the Curie temperature. The consideration of correlated hopping allows one to avoid (for some values of the correlated hopping parameters) overestimation of the Curie temperature and to obtain values which are close to the experimentally observed ones (in this connection see also [15], where the correlated hopping is not taken into account). Let us assume (as in the Ref. 15) 


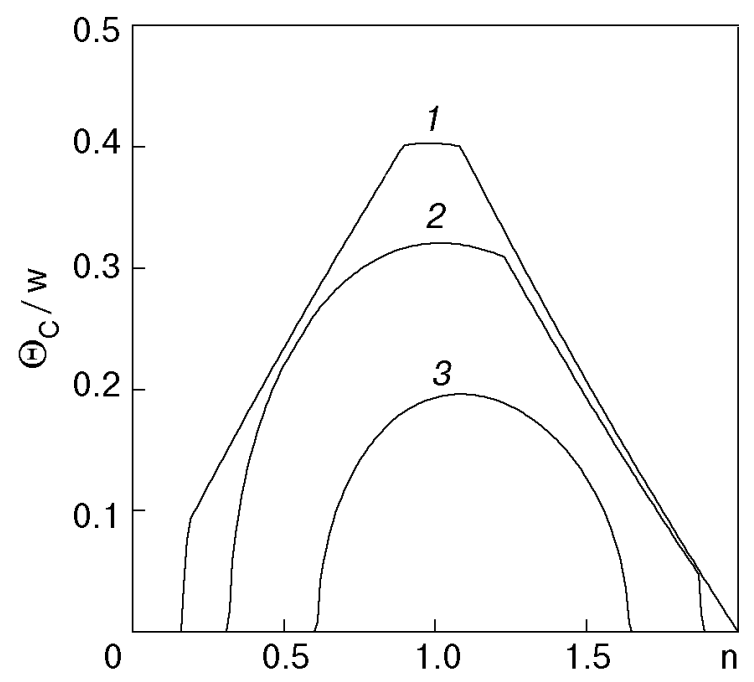

Fig. 3. Curie temperature as a function of $n$ for $U / w=1 \quad$ and $\tau_{1}=0.05, \quad \tau_{2}=0.1 . \quad z J / w=0.4 \quad(1)$; $z J / w=0.5(2), \quad z J / w==0.6$ (3).

that the bandwidth of the $\varepsilon_{q}$ states in $3 d$ ferromagnetic transition metals is approximately $2 \mathrm{eV}$. In our work the band filling $n=1.2$ corresponds to Fe and for the values of correlated hopping $\tau_{1}=0.15$, $\tau_{2}=0.2$ our theory would predict values of the Curie temperature between 1000 and $1600 \mathrm{~K}$, depending on the values of the intra-atomic Coulomb repulsion and exchange interaction (these parameters are varied in the ranges from 0 to 0.4 and from 0.14 to 0.37 , respectively; the larger the value of the parameter $U / w$, the smaller the value of parameter $z J / w$ that is needed). The value of the Curie temperature for some fixed values of the intra-atomic Coulomb interaction and interatomic exchange interaction agrees with the experimental data even quantitatively.

In Fig. 3 the concentration dependence of the Curie temperature is plotted for various values of the exchange interaction. The peculiarity of this dependence is the lowering of the Curie temperature with increasing carrier concentration. Besides, taking into account the correlated hopping causes asymmetry of the curve with respect to half-filling, which allows one to explain qualitatively the larger Curie temperature in Co as compared to Fe. Note that this fact could not be explained in Ref. 15 without further comments (in particular, taking into account peculiarities of the density of states). On the basis of the expression obtained for the Curie temperature one can explain the peculiarities of the Curie temperature behavior in binary alloys of transition metals [26]. It is reasonable to interpret in the framework of our theory to the paramag-

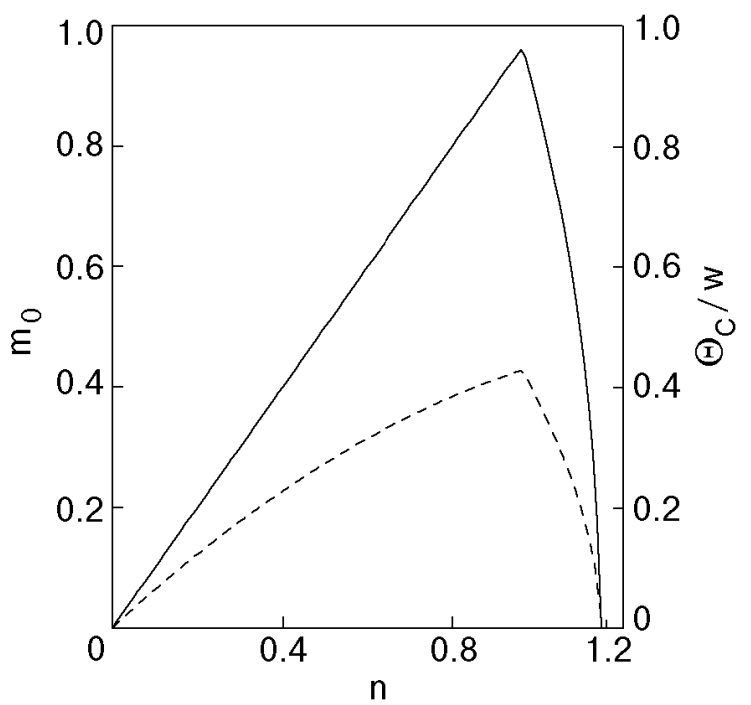

Fig. 4. Dependences of the magnetization (solid curve) and Curie temperature (dashed curve) on electron concentration $n$ for $U / w=1.5, \quad \tau_{1}=0, \quad \tau_{2}=0.8$ and $z J / w=0.2$.

net-ferromagnet transition in metallic phase with increasing temperature for the nonstoichiometric chalcogenide chrome spinel $\mathrm{Cr}_{0.5} \mathrm{Fe}_{0.5} \mathrm{~S}$ (where the Curie temperature is of the order of $1000 \mathrm{~K}$ ) [27].

In principle, our theory allows one to obtain the concentration dependences of the magnetization and Curie temperature, which are similar those observed experimentally in the compounds $\mathrm{Fe}_{1-x} \mathrm{Co}_{x} \mathrm{~S}_{2}$ and $\mathrm{Co}_{1-x} \mathrm{Ni}_{x} \mathrm{~S}_{2}$ with a change of electron concentration in the $3 d$ band [28]. In these crystals the same subsystem of electrons is responsible both for conductivity and for the formation of localized magnetic moments. Although these compounds should be described in the framework of a doubly orbitally degenerate model, nevertheless for some values of the model parameters it is also possible to obtain in terms of a single-band model the concentration dependences of the Curie temperature and magnetic moment of the system (see Fig. 4) in qualitative agreement with the experimentally observed ones [28]. The plotted curves show that for the choosen model parameters the values of the above mentioned quantities are reproduced accurately.

The influence of the model parameters on the critical temperature is illustrated in the next figures. In Figs. 5 and 6 the dependences of the Curie temperature on the intra-atomic Coulomb interaction parameter are plotted at half-filling (Fig. 5) and various values of the band filling (Fig. 6); the values of exchange integral and correlated hopping are fixed. The plotted curves have a peculiarity: one can distinquish an area of sharp increase of the Curie temperature with increase of the parameter 


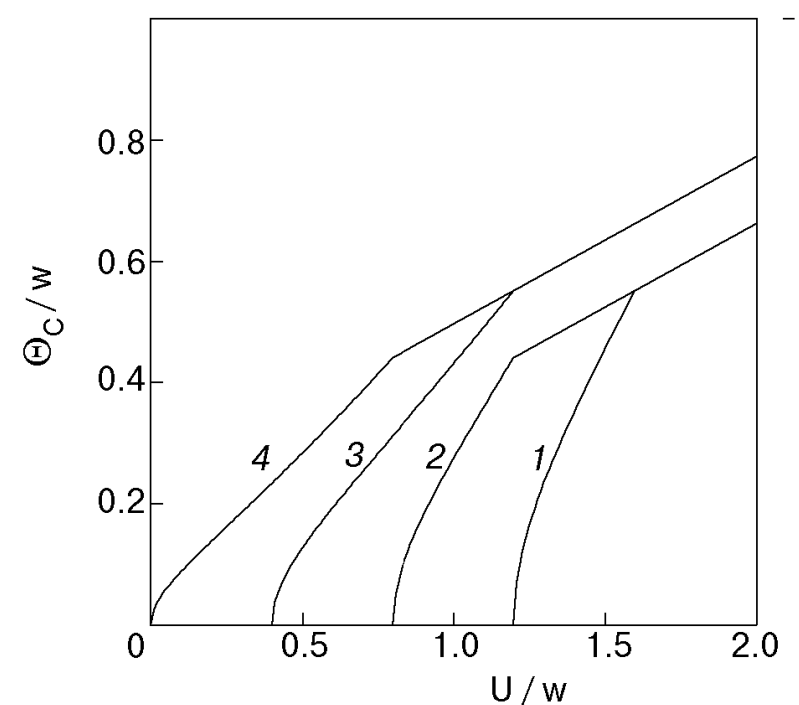

Fig. 5. Curie temperature as a function of $U / w$ at halffilling. $z J / w=0.4, \quad \tau_{1}=\tau_{2}=0 \quad(1) ; z J / w=0.4, \tau_{1}=$ $=\tau_{2}=0.1(2) ; z J / w=0.8, \tau_{1}=\tau_{2}=0(3) ; z J / w=0.8$, $\tau_{1}=\tau_{2}=0.1(4)$.

$U / w$ (these values of the model parameters correspond to partial spin polarization of the system) and an area where the Curie temperature changes in proportion to $U / w$ (these values correspond to full spin polarization of the system). Note that increase of the interatomic exchange interaction leads to extension of the partially polarized ferromagnetic area and also to a decrease of the critical value of $U$ required for the development of saturated ferromagnetism. The peculiarity of the dependence of the Curie temperature on $U / w$ is the increase of the

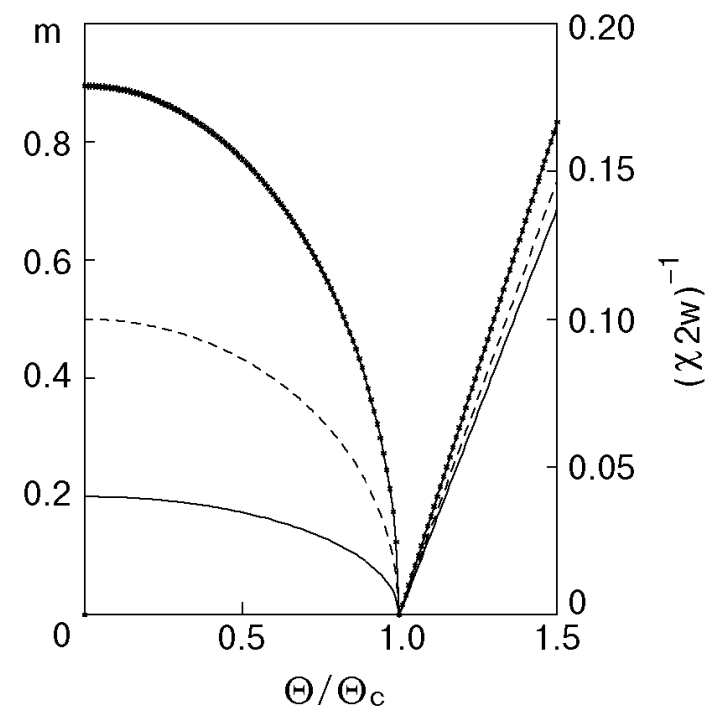

Fig. 7. Dependences of the magnetization and inverse magnetic susceptibility on reduced temperature for $U / w=1$, $z J / w=0.5, \tau_{1}=0.05$ and $\tau_{2}=0.1$; the upper, middle and lower curves correspond to the case $n=1, n=0.5$ and $n=0.2$, respectively.

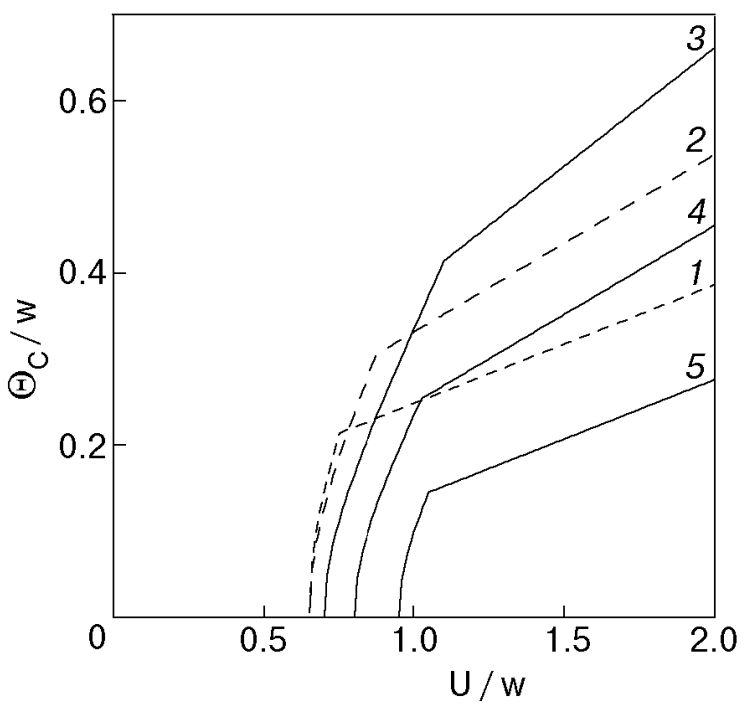

Fig. 6. Curie temperature as a function of $U / w$ at $z J / w=0.4, \tau_{1}=0.05, \tau_{2}=0.2$ and various band fillings. 1, 2, 3, 4 and $5-n=0.5,0.75,1,1.25$ and 1.5, respectively.

critical value of $U / w$ with increasing $n$. Note also that the change of this critical value (for chosen values of correlated hopping) in the case $n>1$ and with increasing electron concentration is more pronounced than in the case $n<1$. It is also interesting that, depending on the magnitude of $U / w$, the system with electron concentration $n<1$ can have a larger value of the Curie temperature than the system with $n>1$. However, starting from the critical point for the intra-atomic Coulomb interaction the situation becomes the opposite: the Curie temperature is larger for the system with $n>1$.

Next consider the behavior of the magnetization of the system with change of temperature. To obtain the temperature dependence of the magnetization $m$ let us use the assumption (as in Ref. 15), that Eq. (17) is also valid for nonzero magnetization and for temperatures lower than the critical $\Theta_{C}$. Then, using (18) one can obtain the equation

$$
\begin{aligned}
& m=\frac{2 \pi}{\sqrt{3}}\left\{\left(\frac{\Theta_{C}}{2 w}\right)^{2} \frac{1}{\left(\alpha^{*}\right)^{2}}-\frac{1}{2}\left(\frac{\Theta}{2 w}\right)^{2} \times\right. \\
& \left.\times\left[\frac{1}{\left(\alpha^{*}+\tau_{2} m\right)^{2}}+\frac{1}{\left(\alpha^{*}-\tau_{2} m\right)^{2}}\right]\right\}^{1 / 2} .
\end{aligned}
$$

The results of numerical calculations for $m$ are plotted in Fig. 7. It should be noted that if the correlated hopping is not taken into account, the expression (22) gives the analytical result of [15]. In the band limit the last expression reproduces the 
result of [29], where a similar treatment is applied to the completely itinerant carriers.

To find the magnetic susceptibility let us take the derivative of the magnetization (8) with respect to the magnetic field,

$$
\begin{gathered}
\chi(\Theta)=\left.\frac{\partial m(h)}{\partial h}\right|_{h \rightarrow 0, m \rightarrow 0}= \\
=\frac{1}{2 w} \frac{1}{\alpha^{*}-U / 2 w-z J / 2 w+2 \tau(n-1)} .
\end{gathered}
$$

Using (17) for temperatures which are close to $\Theta_{C}$ one can obtain

$$
\chi(\Theta)=\frac{6 w\left[U / 2 w+z J / 2 w-2 \tau_{2}(n-1)\right]^{2}}{2 \pi^{2} \Theta_{C}\left(\Theta-\Theta_{C}\right) z J / w} .
$$

In Fig. 7 the temperature dependences of the magnetization and inverse magnetic susceptibility are plotted. A similar plot was obtained for the transition metals in Ref. 30 using the dynamical mean-field theory combined with the local density approximation. For temperatures higher than the Curie temperature the magnetic susceptibility demonstrates Curie-Weiss-like behavior.

\section{Conclusions}

In this paper the ferromagnetic solution in a single-band generalized Hubbard model is derived. The peculiarity of the model is the inclusion in the Hubbard Hamiltonian the interatomic exchange interaction and electron-electron interactions which describe the influence of occupancy of sites on the hopping process. The physical mechanism which leads to realization of the ferromagnetic state is a shift of the electron subband centers caused by the exchange interaction and correlated hopping; in addition, the band narrowing due to correlated hopping is also important.

Taking the correlated hopping into account leads to asymmetry of the cases $n<1$ and $n>1$ for the consideration of ferromagnetism in this model. An increase of the correlated hopping parameter $\tau_{1}$ leads to a shift of the ferromagnetic area to a region of larger electron concentration $n$, while an increase of the correlated hopping parameter $\tau_{2}$ causes a shift to smaller electron concentration $n$. An important consequence of this study is the conclusion that, for the realization of ferromagnetism the case of a more than half-filled band is more favorable (this is the case of transition metals and their alloys) than the case of $n<1$.
The concentration dependence of the groundstate magnetization $m_{0}$ qualitatively agrees with the experimental data for the $3 d$ transition metals and their alloys; in particular, our results can explain Slater-Pouling's curves [26] for the binary ferromagnetic alloys of the transition metals $\mathrm{Fe}, \mathrm{Co}$, and Ni with other $3 d$ metals.

Taking into account the correlated hopping in the calculation of the Curie temperature allows one to obtain values which agree with the experimentally observed ones. The calculated Curie temperature is characterized by peculiarities of the concentration dependence; in particular, asymmetry (in consequence of taking correlated hopping into account) with respect to band half-filling. This result agrees with the experimentally observed values of the critical temperature in the ferromagnetic transition metals. Besides, our results qualitatively reproduce the a typical concentration dependence of the Curie temperature in the systems $\mathrm{Fe}_{1-x} \mathrm{Co}_{x} \mathrm{~S}_{2}$ and $\mathrm{Co}_{1-x} \mathrm{Ni}_{x} \mathrm{~S}_{2}$ [28].

In conclusion, the correct taking into account of the above-mentioned matrix elements of electronelectron interactions allows one to explain some peculiarities of the ferromagnetic properties for transition metal and their alloys and compounds both in the case of the ground state and at nonzero temperatures.

1. J. Hubbard, Proc. R. Soc. A276, 238 (1963).

2. J. Kanamori, Prog. Theor. Phys. 30, 275 (1963).

3. M. C. Gutzwiller, Phys. Rev. Lett. 10, 159 (1963).

4. H. Tasaki, Progr. Theor. Phys. 99, 489 (1998).

5. D. Vollhardt, N. Blumer, K. Held, M. Kollar, J. Schlipf, M. Ulmke, and J. Wahle, Adv. Solid State Phys. 38, 383 (1999).

6. V. Yu. Irkhin and Yu. P. Irkhin, Electronic structure and physical properties of $d$ - and f-transition metals and their compounds, cond-mat/9812072.

7. D. Vollhardt, N. Blumer, K. Held, M. Kollar, J. Schlipf, and M. Ulmke, Z. Phys. B103, 283 (1997).

8. J. Wahle, N. Blumer, J. Schlipf, K. Held, and D. Vollhardt. Phys. Rev. B58, 12749 (1998).

9. M. Kollar and D. Vollhardt, Phys. Rev. B63, 045107 (2001).

10. H.-Q. Nie and W.-Y. Zhou, Phys. Rev. B55, 59 (1997).

11. T. Herrman and W. Nolting, J. Magn. Magn. Mater. 170, 253 (1997).

12. M. Potthoff, T. Herrman, and W. Nolting, Phys. Status Solidi B210, 199 (1997).

13. J. C. Amadon and J. E. Hirsch, Phys. Rev. B54, 6364 (1996).

14. J. E. Hirsch, Phys. Rev. B40, 2354 (1989).

15. J. E. Hirsch, Phys. Rev. B40, 9061 (1989).

16. J. E. Hirsch, Phys. Rev. B59, 6256 (1999). 
17. E. C. Stoner, Proc. R. Soc. A165, 372 (1938).

18. E. P. Wohlfarth, Philos. Mag. 42, 374 (1951).

19. V. Ivanov, Ukr. Phys. J. 36, 751 (1991).

20. L. Didukh, J. of Phys. Stud. 1, 241 (1997), (in Ukrainian).

21. L. Didukh, O. Kramar, and Yu. Skorenkyy, J. Phys. Condens. Matter 4, 101 (2001).

22. L. Didukh, O. Kramar, and Yu. Skorenkyy, Phys. Status Solidi B (2002), in press (cond-mat /0012402).

23. L. Didukh, Sov. Phys. Solid State 19, 711 (1977).

24. L. Didukh, J. Phys. Condens. Matter 1, 125 (1998).

25. N. W. Ashcroft and N. D. Mermin, Solid State Phys., Holt, Rinehart and Winston, New York (1975).
26. F. Gautier, in: Magnetism of Metals and Alloys, M. Cyrot (ed.), North-Holland, Amsterdam (1982).

27. G. V. Loseva, S. G. Ovchinnikov, and G. A. Petrakovsky, Metal-Insulator Transition in Sulphides of $3 d$ Metals, Nauka, Novosibirsk (1983), (in Russian).

28. H. S. Jarrett, W. H. Cloud, R. J. Bouchard, S. R. Butler, C. G. Frederick, and J. L. Gilson, Phys. Rev. Lett. 21, 617 (1965).

29. J. A. Blanco and J. Pisonero, Eur. J. Phys. B20, 289 (1998).

30. A. T. Lichtenstein and M. I. Katsnelson, condmat $/ 0102297$. 